\title{
Comparative genomics reveals Cyclospora cayetanensis possesses coccidia-like metabolism and invasion components but unique surface antigens
}

Shiyou Liu' ${ }^{1,2}$, Lin Wang ${ }^{1}$, Huajun Zheng ${ }^{3}$, Zhixiao Xu' ${ }^{1}$, Dawn M. Roellig ${ }^{2}, \mathrm{Na} \mathrm{Li}^{1,2}$, Michael A. Frace $^{4}$, Kevin Tang ${ }^{4}$, Michael J. Arrowood ${ }^{2}$, Delynn M. Moss', Longxian Zhang ${ }^{5}$, Yaoyu Feng ${ }^{1 *}$ and Lihua Xiao ${ }^{2^{*}}$

\begin{abstract}
Background: Cyclospora cayetanensis is an apicomplexan that causes diarrhea in humans. The investigation of foodborne outbreaks of cyclosporiasis has been hampered by a lack of genetic data and poor understanding of pathogen biology. In this study we sequenced the genome of C. cayetanensis and inferred its metabolism and invasion components based on comparative genomic analysis.

Results: The genome organization, metabolic capabilities and potential invasion mechanism of $C$. cayetanensis are very similar to those of Eimeria tenella. Propanoyl-CoA degradation, GPI anchor biosynthesis, and N-glycosylation are some apparent metabolic differences between C. cayetanensis and E. tenella. Unlike Eimeria spp., there are no active LTRretrotransposons identified in C. cayetanensis. The similar repertoire of host cell invasion-related proteins possessed by all coccidia suggests that $C$. cayetanensis has an invasion process similar to the one in $T$. gondii and $E$. tenella. However, the significant reduction in the number of identifiable rhoptry protein kinases, phosphatases and serine protease inhibitors indicates that monoxenous coccidia, especially C. cayetanensis, have limited capabilities or use a different system to regulate host cell nuclear activities. C. cayetanensis does not possess any cluster of genes encoding the TA4-type SAG surface antigens seen in E. tenella, and may use a different family of surface antigens in initial host cell interactions.

Conclusions: Our findings indicate that C. cayetanensis possesses coccidia-like metabolism and invasion components but unique surface antigens. Amino acid metabolism and post-translation modifications of proteins are some major differences between $C$. cayetanensis and other apicomplexans. The whole genome sequence data of $C$. cayetanensis improve our understanding of the biology and evolution of this major foodborne pathogen and facilitate the development of intervention measures and advanced diagnostic tools.
\end{abstract}

Keywords: Cyclospora, Genomics, Genome, Genetics, Evolution, Apicomplexan

\section{Background}

Cyclospora cayetanensis is an emerging apicomplexan parasite related to Eimeria spp. [1]. After ingestion of food or water contaminated by oocysts, humans develop watery diarrhea, nausea and abdominal pain. In industrialized

\footnotetext{
* Correspondence: yyfeng@ecust.edu.cn; Ixiao@cdc.gov

${ }^{1}$ State Key Laboratory of Bioreactor Engineering, School of Resources and Environmental Engineering, East China University of Science and Technology, Shanghai 200237, China

${ }^{2}$ Division of Foodborne, Waterborne, and Environmental Diseases, Centers for Disease Control and Prevention, Atlanta, GA 30333, USA

Full list of author information is available at the end of the article
}

nations cyclosporiasis is often associated with travel to developing countries or outbreaks due to consumption of imported fresh produce [1, 2]. Since 2013, large multistate outbreaks of cyclosporiasis have occurred yearly in the United States and Canada, but outbreak investigations have been hampered by the lack of molecular diagnostic tools for trace-back studies [3] (http://www.cdc.gov/parasites/cyclosporiasis/outbreaks/2015/index.html).

The life cycle of $C$. cayetanensis is typical of monoxenous coccidia, which complete asexual and sexual development within a single host. Similar to Eimeria spp., C. cayetanensis 
probably has strict host specificity, infecting only enterocytes of humans. In contrast, another well-studied coccidian parasite, Toxoplasma gondii, has a heteroxenous life cycle, infecting not only enterocytes of its feline definitive hosts but also multiple tissues of various intermediate hosts, including humans [4]. The molecular determinants of host specificity and tissue tropism in apicomplexan parasites are poorly understood. Nevertheless, the host cell invasion mechanism of $T$. gondii has been studied extensively. Three essential secretory organelles, including micronemes and rhoptries of the apical complex and dense granules, are involved in the invasion process [5]. Before host cell invasion, apicomplexan sporozoites move across substrates by gliding, which is powered by an actinmyosin motor. The invasion begins with the secretion of several groups of proteins from micronemes, such as the apical membrane antigen 1 (AMA1) and rhoptry neck proteins (RONs), such as RON2, RON4 and RON5, forming a moving junction that is attached to the host cell cytoskeleton. This leads to the formation of numerous host-pathogen adhesion complexes consisting of microneme proteins (MICs) and surface antigens [6]. The parasite then moves across host membranes and develops a parasitophorous vacuole (PV) inside the host cell, where it grows and replicates. To evade the host immune system and survive in the intracellular environment, another large group of rhoptry proteins (ROPs) are delivered to the periplasmic surface of the PV and host cell nucleus, modulating host cell signaling pathways and gene expression $[7,8]$. Some proteins secreted from dense granules (GRAs) are also involved in the regulation of host cell nuclear activities [9].

Few data exist on genetics of $C$. cayetanensis. To generate much needed sequence data and improve our understanding of its biology, we sequenced the genome of an isolate of $C$. cayetanensis and conducted a comparative genomic analysis. The results show that $C$. cayetanensis and $E$. tenella have similar genomic features and metabolic capabilities. They probably use a host cell invasion system similar to that in $T$. gondii, but a divergent system in modulating host cell signaling pathways. The specific surface antigens possessed by different coccidia may be the primary determinants for their host specificity.

\section{Results}

\section{Genome sequencing and general features}

We obtained 120.9 million of 100-bp paired-end reads from Illumina sequencing and 960,078 reads of 400-450 bp from Roche GS-FLX 454 sequencing, yielding over 200-fold coverage of the genome. A total of 4811 contigs with an overall length of 46,816,962 bp were generated in the de novo assembly of sequences (Additional file 1: Figure S1). After BLASTN analysis to eliminate contaminants from bacteria, Archaea, or host DNA, we obtained a draft genome of $C$. cayetanensis with a total length of $44,034,550 \mathrm{bp}$, a mean contig length of $19,170 \mathrm{bp}$, and an N50 contig of 61,202 bp (Additional file 2: Table S1). The genome of $C$. cayetanensis is slightly smaller than genomes of T. gondii and E. tenella (Table 1). The completeness of the draft genome of $C$. cayetanensis was estimated by using the BUSCO software (Additional file 3: Table S2). Altogether, $74.4 \%$ of the core eukaryotic protein-encoding genes were covered by the genome of $C$. cayetanensis, which is comparable to that of whole genome sequences from T. gondii (85.1 \%) and E. tenella (68.1\%). It has a gene density that is similar to that of E. tenella and $T$. gondii, but lower than that seen in some other apicomplexan parasites. In BLASTN analysis, we have identified the full mitochondrial and apicoplast genomes of C. cayetanensis [10].

The alternation of repeat-rich and repeat-poor regions, which was reported for Eimeria spp. [11], was also detected in the $C$. cayetanensis genome (Fig. 1). In addition, the most common short tandem repeats (STRs) are also "CAG" motif and variations of it, as seen in Eimeria genomes [11]. There are 87 putative long terminal repeat (LTR) retrotransposons in the C. cayetanensis genome (Additional file 4: Figure S2). The length of putative LTRs in C. cayetanensis varies from 106 to $996 \mathrm{bp}$ with an average of $337 \mathrm{bp}$, and the sequence similarity between upstream and downstream LTRs of each retrotransposon varies from $85.0 \%$ to $98.6 \%$. Cluster analysis showed that they could be divided into 44 types based on sequence identities. Unlike Eimeria spp., whose LTR-retrotransposons belong to chromoviruses, neither the chromodomain nor the functional domain of reverse transcriptases was identified in LTR-retrotransposons of $C$. cayetanensis. In a phylogenetic analysis, a representative LTR-retrotransposon sequence from $C$. cayetanensis was placed outside the clade formed by chromoviruses (Additional file 5: Figure S3).

\section{Gene content}

There are 144 predicted tRNA genes in the $C$. cayetanensis genome, which is slightly fewer than 174 in $T$. gondii but much higher than in other apicomplexans. We identified 11 rRNA genes in the draft genome of $C$. cayetanensis (Table 1).The $C$. cayetanensis genome may encode as many as 7457 proteins. Among them, 538 proteins have signal peptides (105 of them target the apicoplast), 1247 had one or more transmembrane regions, and 225 had a GPIanchor attachment site. These numbers are similar to those in E. tenella and T. gondii (Table 1).

OrthoMCL and BLASTP were used to identify the closest orthologs of the predicted proteins of $C$. cayetanensis. The majority of orthologs were from alveolates $(n=6024)$, but several were from other organisms $(n=34)$ (Fig. 2a). All orthologs of bacterial genes found in C. cayetanensis are also present in other apicomplexans, implying a possible origin through lateral gene transfer. By Pfam 
Table 1 Comparison of genomic features of Cyclospora cayetanensis (Ccay) and other apicomplexan parasites ${ }^{a}$

\begin{tabular}{|c|c|c|c|c|c|c|}
\hline Category & Cpar & Pfal & Bbov & Tgon & Eten & Ccay \\
\hline No. of chromosomes & 8 & 14 & 4 & 14 & 14 & - \\
\hline Total length of assembly (Mb) & 9.10 & 22.85 & 8.18 & 65.67 & 51.86 & 44.03 \\
\hline No. of super contigs & 8 & 16 & 14 & 2,263 & 4,664 & 2,297 \\
\hline GC content (\%) & 30.3 & 20.0 & 41.5 & 48.5 & 52.5 & 51.8 \\
\hline No. of genes & 3,805 & 5,542 & 3,706 & 8,322 & 8,597 & 7,457 \\
\hline Total length of CDS (Mb) & 6.83 & 12.58 & 5.58 & 20.03 & 13.05 & 11.92 \\
\hline GC content in CDS (\%) & 31.9 & 25.0 & 43.7 & 56.0 & 58.1 & 55.8 \\
\hline Mean length of genes (bp) & 1,720 & 2,271 & 1,506 & 2,407 & 1,518 & 1,599 \\
\hline Gene density (genes/Mb) & 418.1 & 242.5 & 453.1 & 126.7 & 165.8 & 169.4 \\
\hline Percent coding (\%) & 75.0 & 55.1 & 68.2 & 30.5 & 25.2 & 27.1 \\
\hline No. of genes with intron & 163 & 3,055 & 2,241 & 6,729 & 6,563 & 6,358 \\
\hline$\%$ genes with introns & 4.2 & 55.1 & 60.5 & 80.9 & 76.3 & 85.3 \\
\hline No. of tRNA & 45 & 72 & 70 & 174 & - & 144 \\
\hline No. of tRNA ${ }^{\text {Met }}$ & 2 & 2 & 4 & 8 & - & 7 \\
\hline No. of rRNA ${ }^{b}$ & 15 & 28 & - & 420 & 4 & 11 \\
\hline No. of proteins with signal peptide & 397 & 638 & 350 & 759 & 775 & 538 \\
\hline No. of proteins with apicoplast targeting signal & (22) & 189 & 99 & 148 & 182 & 105 \\
\hline No. of proteins with transmembrane domain & 832 & 1,754 & 677 & 1,103 & 1,378 & 1,247 \\
\hline No. of proteins with GPI-anchor & 63 & 62 & 51 & 255 & 371 & 225 \\
\hline Apicoplast genome size (bp) & - & 34,682 & 33,351 & 34,996 & 34,750 & 34,155 \\
\hline Mitochondrial genome size (bp) & - & 5,967 & 6,005 & $\sim 6,000^{c}$ & 6,213 & 6,229 \\
\hline
\end{tabular}

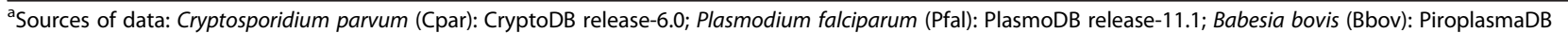
release-5.1; Toxoplasma gondii (Tgon): ToxoDB release-11.0; Eimeria tenella (Eten): ToxoDB release-11.0. Data on proteins with signal peptides, apicoplast targeting signal peptides and GPI-anchors were based on calculations using software specified in Methods. Dashes indicate the lack of data (for $E$. tenella) or the absence of organelles (for C. parvum)

${ }^{\mathrm{b}}$ Based on annotation; actual numbers are greater due to the repetitive nature of the rRNA unit

${ }^{\mathrm{c}}$ Based on Seeber et al. (2014) [72]

searching, there is a large group ( 1020) of functional domains shared by apicomplexans and a smaller group ( 546$)$ by coccidia (Fig. 2b). The heteroxenous T. gondii apparently possesses more unique protein domains than the monoxenous E. tenella and C. cayetanensis. Phylogenetic analysis of 100 orthologous protein sequences confirmed the close relatedness of $C$. cayetanensis to $E$. tenella (Fig. 2c).

\section{Carbohydrate and energy metabolism}

Similar to most other apicomplexans, C. cayetanensis depends on carbon metabolism, including glycolysis, tricarboxylic acid (TCA) cycle and pentose phosphate pathways, for energy generation (Table 2, Additional file 6: Table S3). The final product, proton, goes through the electron transport system mediated by a series of membrane-bound mitochondrial enzymes to generate the energy carrier, ATP. The classical NADH dehydrogenase multi-protein complex, complex I, is absent in all apicomplexans, being substituted by an alternative single NADH dehydrogenase [12]. Three other multiple-protein complexes (II-IV) and an ATPase (complex V) are present in C. cayetanensis. As a coccidian parasite, $C$. cayetanensis has the capability to store energy in the form of the red algae-like 'floridean starch', a variant of amylopectin synthesized by using UDP-Glc (glucose) rather than ADP-Glc used in green algae and land plants [13]. All coccidia have the ability to concatenate UDP-Glc into 1,3-beta-glucans and also likely have a galactose metabolism. E. tenella and C. cayetanensis have the unique ability to reversely produce mannitol from fructose. A similar pathway may be present in Cryptosporidium spp., although it utilizes mannose rather than fructose [12]. The amino and nucleotide sugars, such as UDP-Glc, UDPGlcNAc (N-acetylglucosamine), and GDP-Man (mannose), are critical resources for the glycosylation of self-generated proteins [12]. All apicomplexans possess this pathway and are able to synthesize these nucleotide sugars. Only coccidia have the enzyme to convert UDP-Glc and UDP-Gal (galactose) in both directions. The reverse conversion between GDP-Man and GDP-Fuc (fucose) is present only in P. falciparum and coccidia. Cryptosporidium spp. are able to convert UDP-Glc into UDP-GlcA (glucuronate) and then into UDP-Xyl (xylose). 


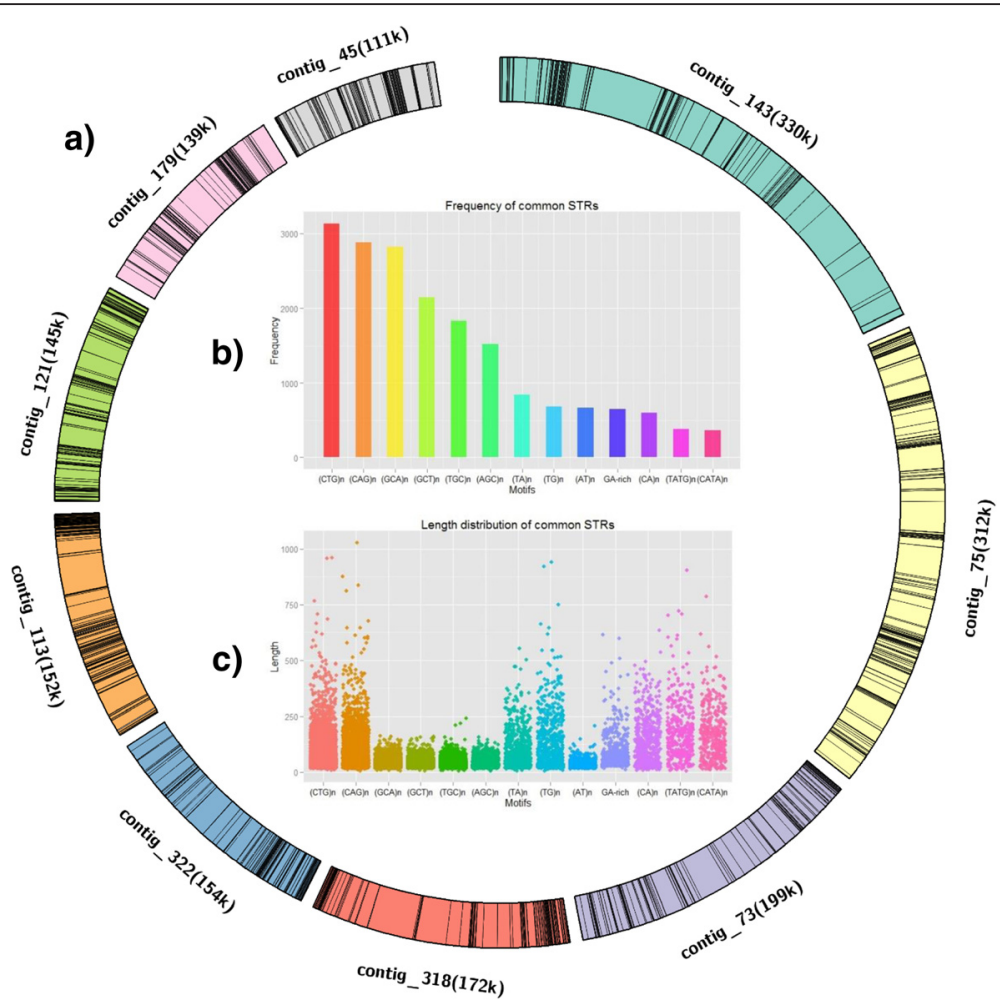

Fig. 1 Alternating regions of repeat-rich and repeat-poor sequences and common short tandem repeats (STRs) in the Cyclospora cayetanensis genome. A total of 1,700 STRs were detected in the C. cayetanensis genome a Alternating pattern of repeat-rich and repeat-poor within several large contigs of the C. cayetanensis genome. The black bands represent STRs. $\mathbf{b}$ Distribution of STRs. The most common STRs are the "CAG" motif and its variations, like seen in Eimeria spp. c Jitter plot showing length distribution of common STRs

Within the pyruvate metabolism, C. cayetanensis and E. tenella possess neither the phosphoenolpyruvate (PEP) carboxylase utilized by P. falciparum and Cryptosporidium spp. nor the pyruvate carboxylase present in T. gondii [12]. However, a PEP carboxykinase is present in C. cayetanensis, E. tenella and other apicomplexans except Cryptosporidium spp., allowing them to continuously produce oxaloacetate to supplement the TCA cycle. In P. falciparum and $T$. gondii, the glycolysis and TCA cycle are disconnected due to the fact that the pyruvate dehydrogenase complex is localized in apicoplasts rather than mitochondria [14]. In addition to all enzymes needed for the TCA cycle in mitochondria, the aconitase dually targeting the mitochondria and apicoplast and an isoenzyme of isocitrate dehydrogenase (ICDH1) targeting the apicoplast are present in $T$. gondii, suggesting that a partial TCA cycle exists in its apicoplast $[12,15]$. The genes encoding two aconitases, the ortholog of ICDH1, and isoforms of citrate synthases were detected in nuclear genomes of $C$. cayetanensis and E. tenella. Thus, C. cayetanensis probably also possesses a partial TCA pathway in its apicoplast.

Like most other apicomplexan parasites, $C$. cayetanensis probably uses the pentose phosphate pathway to produce de novo phosphoribosyl pyrophosphate (PRPP), which is involved in pyrimidine biosynthesis. A ribokinase is present only in T. gondii, E. tenella and C. cayetanensis, suggesting that only coccidia are able to salvage ribose from the host in addition to synthesizing it de novo. Compared to $P$. falciparum and T. gondii, the deoxyribose phosphate aldolase for deoxyribose catalysis is absent in both E. tenella and C. cayetanensis. Another important intermediate within the pentose phosphate pathway, erythrose-4-phosphate, is the substrate in biosynthesis of shikimate as well as folate, which is eventually converted into tetrahydrofolate (THF) and methylene-THF. These two folates are essential for nucleotide conversion and amino acid conversion, respectively. In addition to the de novo synthesis of folates, apicomplexan parasites can transport folic acid from the extracellular environment using specific cytosol membrane transporter proteins (Table 3). Furthermore, T. gondii possesses two extended sub-pathways for folate metabolism: 1) the biosynthesis of dihydrobiopterin and tetrahydrobiopterin, which can provide hydroxyl for converting phenylalanine to tyrosine; and 2) the biosynthesis of molybdopterin, the cofactor for sulfite oxidation [12]. None of these enzymes or proteins were identified in C. cayetanensis. 
a)

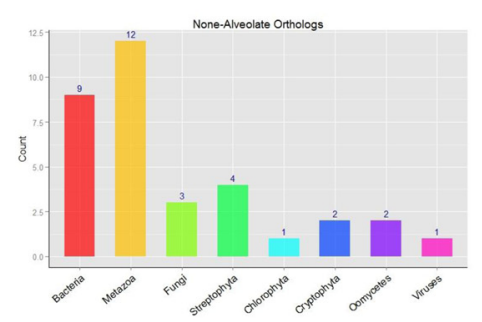

c)

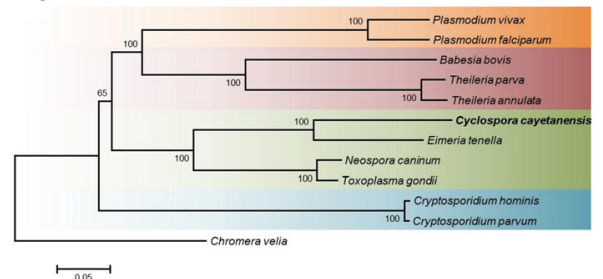

b)

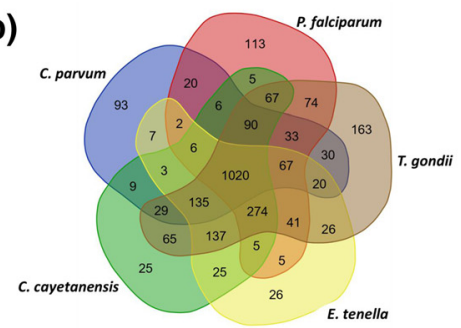

d)

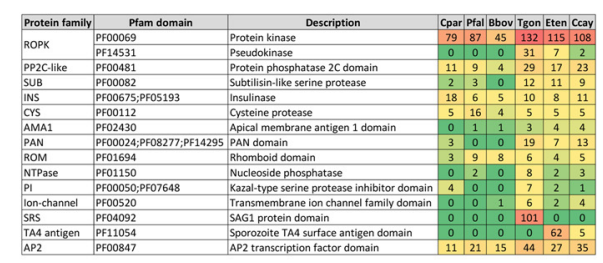

Fig. 2 Orthologs in the predicated proteome of Cyclospora cayetanensis. a In addition to alveolates, a few of the orthologs of C. cayetanensis are from other organisms, probably resulted from lateral gene transfers. b Functional protein domains shared by apicomplexan parasites Cryptosporidium parvum, Plasmodium falciparum, Toxoplasma gondii, Eimeria tenella and C. cayetanensis. c Phylogenetic relationship of C. cayetanensis and other common apicomplexan parasites based on a neighbor-joining analysis of concatenated protein sequences from 100 orthologs; a concatenated sequence from the free-living photosynthetic chromerid, Chromera velia was used to root the tree. The maximum composite likelihood method was used in the calculation of genetic distances. Numbers on branches are percent bootstrap values $>50$ from 1,000 replications. $\mathbf{d}$ Comparison of major protein families potentially involved in host cell invasion among common apicomplexan parasites. Taxa name abbreviations: Cryptosporidium parvum (Cpar); Plasmodium falciparum (Pfal); Babesia bovis (Bbov); Toxoplasma gondii (Tgon); Eimeria tenella (Eten); Cyclospora cayetanensis (Ccay)

Fatty acid biosynthesis in apicomplexans is thought to occur in the apicoplast through type II fatty acid synthases encoded in the nuclear genome [14]. Some apicomplexans also possess the prokaryotic type I fatty acid synthase in the cytosol to elongate short-chain fatty acids salvaged from the host [14]. The genes coding both types of fatty acid synthases are present in the C. cayetanensis genome, similar to $E$. tenella and T. gondii (Table 2, Additional file 6: Table S3). Most apicomplexans synthesize isoprenoids in the apicoplast through a bacteria-type DOXP pathway utilizing phosphoenol pyruvate and dihydroxyacetone phosphate [14]. The complete set of enzymes involved in isoprenoid biosynthesis including the apicoplast glyceraldehyde-3phosphate dehydrogenase isoenzyme characterized in $T$. gondii [16] was detected in C. cayetanensis.

\section{Amino acids metabolism}

Similar to T. gondii and E. tenella, C. cayetanensis can synthesize alanine from pyruvate while other apicomplexans have to salvage it from the host (Table 2, Additional file 6: Table S3). Except for Cryptosporidium spp., all apicomplexans including C. cayetanensis can utilize nitrite or nitrate transported from the host to synthesize glutamate, which can be converted into glutamine through glutamine synthetase in coccidia and P. falciparum. Except for Cryptosporidium spp., all apicomplexans can reversely convert oxaloacetate and glutamate to aspartate. Only coccidia have the ability to generate proline from glutamate as in humans, the enzymes for producing serine de novo from glycerate or glycerol phosphate, and the inter-converting serine into cysteine as in humans and animals.

Among apicomplexans, only $T$. gondii possesses the capacity of biosynthesis of lysine and threonine from aspartate, whilst there is only a putative threonine synthase in C. cayetanensis. Another important amino acid in apicomplexans, methionine, is probably salvaged from the host, and can be converted to homocysteine, the substrate for the biosynthesis of cysteine. In P. falciparum and $T$. gondii, homocysteine can be potentially recycled into methionine [12]. Due to the lack of an arginase, no coccidia can synthesize ornithine, the substrate for the biosynthesis of polyamines from arginine, as in P. falciparum. However, coccidia have the capability to convert proline into ornithine. Only $P$. falciparum can synthesize polyamines, spermidine and spermine through putrescine (http://mpmp.huji.ac.il). However, all coccidia can probably synthesize putrescine reversely from spermine salvaged from the host.

No apicomplexans are able to synthesize aromatic amino acids de novo; they have to salvage them from the host through an amino acid transporter embedded in the plasma membrane [12]. C. cayetanensis and E. tenella, however, each has only one amino acid transporter, compared with 10 in Cryptosporidium spp. and 6 in T. gondii (Table 3). Some of the $>20 \mathrm{ABC}$ transporters present in each genome could be responsible for the 
Table 2 Comparison of some essential metabolic pathways among common apicomplexan parasites ${ }^{a}$

\begin{tabular}{|c|c|c|c|c|c|c|c|}
\hline Category & Metabolic pathway & Cpar & Pfal & Bbov & Tgon & Eten & Ccay \\
\hline \multirow[t]{26}{*}{ Carbohydrate and energy metabolism } & Glycolysis & + & + & + & + & + & + \\
\hline & $\begin{array}{l}\text { Degradation of propionyl-CoA into pyruvate and } \\
\text { succinate }\end{array}$ & - & - & - & + & - & + \\
\hline & TCA cycle & - & + & + & + & + & + \\
\hline & Pentose phosphate pathway & - & + & + & + & + & + \\
\hline & Shikimate biosynthesis & - & + & - & + & + & + \\
\hline & Folate biosynthesis & - & + & - & + & + & + \\
\hline & $\begin{array}{l}\text { Synthesis of tetrahydrobiopterin/dihydrobiopterin/ } \\
\text { molybdopterin }\end{array}$ & - & - & - & + & - & - \\
\hline & Galactose metabolism & - & - & - & + & + & + \\
\hline & Synthesis of starch & + & - & - & + & + & + \\
\hline & Synthesis of trehalose & + & - & + & + & + & + \\
\hline & Synthesis of 1,3-beta-glucan & - & - & - & + & + & + \\
\hline & Conversion between UDP-Glc and UDP-Gal & + & - & - & + & + & + \\
\hline & Conversion between GDP-Man and GDP-Fuc & - & + & - & + & + & + \\
\hline & Conversion of UDP-Glc to UDP-GlcA then to UDP-Xyl & + & - & - & - & - & - \\
\hline & Synthesis of mannitol from mannose or fructose & + & - & - & - & + & + \\
\hline & Fatty acid biosynthesis in cytosol (FAS I) & + & - & - & + & + & + \\
\hline & Fatty acid biosynthesis in apicoplast (FAS II) & - & + & - & + & + & + \\
\hline & Fatty acid degradation & - & - & - & + & + & + \\
\hline & Oxidative phosphorylation (NADH dehydrogenase) & + & + & + & + & + & + \\
\hline & Oxidative phosphorylation (Complex II) & - & + & + & + & + & + \\
\hline & Oxidative phosphorylation (Complex III) & - & + & + & + & + & + \\
\hline & Oxidative phosphorylation (Complex IV) & - & + & + & + & + & + \\
\hline & F-ATPase & 2 subunits & + & + & + & + & + \\
\hline & V-ATPase & + & + & + & + & + & + \\
\hline & Glyoxalase metabolism producing D-lactate & - & + & + & + & + & + \\
\hline & Synthesis of isoprene (MEP/DOXP) & - & + & + & + & + & + \\
\hline \multirow[t]{2}{*}{ Nucleotide metabolism } & Synthesis of purine rings de novo & - & - & - & - & - & - \\
\hline & Synthesis of pyrimidine de novo & - & + & + & + & + & + \\
\hline \multirow[t]{13}{*}{ Amino acid metabolism } & Synthesis of alanine from pyruvate & - & - & - & + & + & + \\
\hline & Synthesis of glutamate from nitrite/nitrate & - & + & + & + & + & + \\
\hline & Conversion from glutamate to glutamine & + & + & - & + & + & - \\
\hline & $\begin{array}{l}\text { Synthesis of aspartate from oxaloacetate and } \\
\text { glutamate }\end{array}$ & - & + & + & + & + & + \\
\hline & Conversion from aspartate to asparagine & + & + & - & + & + & + \\
\hline & Conversion from glutamate to proline & + & - & - & + & + & + \\
\hline & $\begin{array}{l}\text { Synthesis of serine from glycerate/glycerol } \\
\text { phosphate }\end{array}$ & - & - & - & + & + & + \\
\hline & Conversion from serine to cysteine & - & - & - & + & + & + \\
\hline & Conversion from serine to glycine & + & + & + & + & + & + \\
\hline & Recycle homocysteine into methionine & - & + & - & + & - & - \\
\hline & Synthesis of lysine from aspartate & - & - & - & + & - & - \\
\hline & Synthesis of threonine from aspartate & - & - & - & + & - & - \\
\hline & Synthesis of ornithine from arginine & - & + & - & - & - & - \\
\hline
\end{tabular}


Table 2 Comparison of some essential metabolic pathways among common apicomplexan parasites ${ }^{\mathrm{a}}$ (Continued)

\begin{tabular}{|c|c|c|c|c|c|c|c|}
\hline & Synthesis of ornithine from proline & - & + & - & + & + & + \\
\hline & Synthesis of polyamine from ornithine & - & + & - & - & - & - \\
\hline & Polyamine pathway backward & + & - & - & + & + & + \\
\hline & Degradation of leucine to acetyl-CoA & - & - & - & + & - & - \\
\hline & Degradation of isoleucine/valine & - & - & - & + & + & + \\
\hline & Aromatic amino acid hydroxylases (AAAH) & - & - & - & + & - & - \\
\hline \multirow[t]{10}{*}{ Vitamin and others } & Synthesis of thiamine (vitamin B1) & - & + & - & - & - & - \\
\hline & Conversion from thiamine to thiamine pyrophosphate (TPP) & - & + & - & + & - & + \\
\hline & Synthesis of FMN/FAD from riboflavin & - & + & + & + & + & + \\
\hline & Synthesis of pyridoxal phosphate (vitamin B6) de novo & - & + & - & + & - & - \\
\hline & Synthesis of $\mathrm{NAD}(\mathrm{P})+$ de novo from nicotinate/nicotinamide & - & + & - & + & + & + \\
\hline & Synthesis of pantothenate from valine & - & - & - & + & + & + \\
\hline & Synthesis of CoA from pantothenate & + & + & + & + & + & + \\
\hline & Synthesis of lipoic acid de novo in apicoplast & - & + & - & + & + & + \\
\hline & Salvage lipoic acid in mitochondria & - & + & + & + & - & + \\
\hline & Synthesis of porphyrin/cytochrome proteins & - & + & - & + & + & + \\
\hline
\end{tabular}

aplus symbol denotes that the essential enzymes for pathways were identified, whereas minus symbol denotes that the essential enzymes for pathways were absent. Only 2 subunits of the F-type ATPase are present in Cryptosporidium parvum. Abbreviation: Cryptosporidium parvum (Cpar); Plasmodium falciparum (Pfal); Babesia bovis (Bbov); Toxoplasma gondii (Tgon); Eimeria tenella (Eten); Cyclospora cayetanensis (Ccay)

Table 3 Putative transporters in common apicomplexan parasites*

\begin{tabular}{|c|c|c|c|c|c|c|c|}
\hline Substrate & Cellular location & Cpar & Pfal & Bbov & Tgon & Eten & Ccay \\
\hline Hexose & & 2 & 2 & 2 & 5 & 5 & 5 \\
\hline Triose phosphate & Plasma/apicoplast membrane & 7 & 4 & 5 & 4 & 1 & 1 \\
\hline Amino acids & Plasma membrane & 10 & 1 & 0 & 6 & 1 & 1 \\
\hline Nucleobase/nucleoside & Plasma membrane & 1 & 4 & 0 & 4 & 3 & 4 \\
\hline Nucleotide-sugar & Plasma membrane & 3 & 1 & 0 & 4 & 1 & 2 \\
\hline Folate/pterine & Plasma membrane & 1 & 2 & 1 & 7 & 4 & 5 \\
\hline Formate/nitrite & & 0 & 1 & 1 & 3 & 2 & 2 \\
\hline GABA (aminobutanoate) & Plasma/mitochondrial membrane & $\mathbf{0}$ & 2 & 1 & 5 & 2 & 2 \\
\hline Acetyl-CoA & & 1 & 1 & 1 & 1 & 1 & 1 \\
\hline Chloride & & 0 & 0 & 0 & 2 & 1 & 1 \\
\hline Inorganic phosphate & & 0 & 1 & 1 & 1 & 1 & 1 \\
\hline Sulfate & & 1 & 1 & 1 & 4 & 2 & 2 \\
\hline Sodium/potassium/calcium & & 2 & 0 & 3 & 9 & 5 & 6 \\
\hline Zinc & & 2 & 2 & 2 & 4 & 3 & 3 \\
\hline Copper & & 1 & 2 & 1 & 3 & 2 & 3 \\
\hline Choline & Plasma membrane & 0 & 1 & 0 & 2 & 1 & 2 \\
\hline Cadmium/zinc/cobalt (efflux) & Plasma membrane & 1 & 1 & 0 & 1 & 1 & 1 \\
\hline Glycerol/water & Plasma membrane & $\mathbf{0}$ & 2 & 0 & 2 & 1 & 2 \\
\hline $\mathrm{ABC}$ transporter** & Plasma membrane & 21 & 16 & 10 & 24 & 25 & 23 \\
\hline Mitochondrial carrier** & Mitochondrial membrane & 9 & 14 & 7 & 21 & 14 & 21 \\
\hline
\end{tabular}

*The detection of putative transporter proteins was based on Pfam search. Abbreviation: Cryptosporidium parvum (Cpar); Plasmodium falciparum (Pfal); Babesia bovis (Bbov); Toxoplasma gondii (Tgon); Eimeria tenella (Eten); Cyclospora cayetanensis (Ccay)

${ }^{* *} \mathrm{ABC}$ transporter and mitochondrial carrier have a broad range of substrates 
salvage of some aromatic amino acids. Within the phenylalanine and tyrosine catabolism pathway, there are two aromatic amino acid hydroxylases in T. gondii, catalyzing the hydroxylation of phenylalanine to synthesize tyrosine and L-DOPA [12]. The genes encoding these enzymes were not detected in $C$. cayetanensis and other apicomplexans. For the catabolism of branched chain amino acids, only $T$. gondii potentially has the ability to generate acetyl-CoA through the degradation of leucine. Compared to $P$. falciparum, which possesses only the early steps of the pathway, coccidia can degrade isoleucine and valine to generate propionylCoA and (R)-methyl-malonyl-CoA, respectively, supplementing intermediates for the TCA cycle [12]. T. gondii and $C$. cayetanensis have the full set of enzymes for the degradation of propionyl-CoA, generating pyruvate and succinate. In addition, $T$. gondii has a pyruvate carboxylase, catalyzing pyruvate to oxaloacetate to make the methyl-citrate cycle a full pathway, similar to bacteria and fungi [12].

\section{Nucleotide metabolism}

No apicomplexans have the ability to synthesize purine rings de novo and have to salvage them from the host (Table 2, Additional file 6: Table S3). There are four homologous genes encoding nucleoside transporters in $C$. cayetanensis (Table 3 ). In addition, the presence of an adenosine kinase (AdK) indicates that adenosine may be the major purine utilized by $C$. cayetanensis, in contrast to the AMP used by P. falciparum $[17,18]$. Like most other apicomplexans except Cryptosporidium spp., C. cayetanensis possesses all the enzymes for synthesizing pyrimidine de novo from aspartate and glutamine, except for the orotate phosphoribosyl transferase that catalyzes the phosphorylation of orotate using PRPP. In line with a parasitic life style, coccidian parasites have a salvage pathway for pyrimidine in addition to its de novo biosynthesis.

\section{Coenzymes, vitamins and other metabolism}

Similar to P. falciparum, T. gondii and E. tenella, C. cayetanensis possesses almost all enzymes needed to synthesize the coenzymes $\mathrm{NAD}^{+}$and $\mathrm{NADP}^{+}$from nicotinate (Table 2, Additional file 6: Table S3). Coccidia can synthesize acyl-chain carrier coenzyme A (CoA) de novo from valine, but other apicomplexans have to salvage pantothenate from the host and convert it into CoA. In apicomplexans, only $P$. falciparum possesses the enzymes synthesizing thiamine from intermediates, whereas other apicomplexan parasites have to salvage it from the host. A single enzyme reaction that catalyzes pyro-phosphorylation of thiamine producing thiamine pyrophosphate, the active form of vitamin B1, is present in P. falciparum, T. gondii and C. cayetanensis. The absence of pyridoxal 5-phosphate (PLP) synthase in $C$. cayetanensis and $E$. tenella suggests that these parasites may have lost the ability to synthesize PLP, a component of vitamin B6, de novo from glutamine. However, the salvage pathways through the phosphorylation of pyridoxal or oxidation of pyridoxine/pyridoxamine phosphate are present in C. cayetanensis [12]. It has been shown that lipoic acid (LA), the critical cofactor for some dehydrogenase complexes, can be synthesized de novo in the apicoplast, or salvaged from the host and utilized in the mitochondrion in $T$. gondii $[12,19]$. The catalytic enzymes involved in the LA metabolism were all detected in C. cayetanensis. E. tenella lacks the enzymes used in mitochondria, but possesses dehydrogenase complexes similar to $T$. gondii and C. cayetanensis, suggesting that this inferred gene loss may not be true.

GPI-anchor, N-glycan, and mucin-type O-glycan biosynthesis Most surface antigens of apicomplexans involved in host cell recognition, interaction or adhesion use a glycosylphosphatidylinositol (GPI) anchor for attachment to the plasma membrane, such as SRS (SAG1-related sequences) proteins of $T$. gondii and TA4-type surface antigens of E. tenella [11]. Two essential mannosyltransferases in the biosynthesis of the GPI-anchor, PIG-V and PIG-B, were not identified in C. cayetanensis and E. tenella (Fig. 3a). In addition, the modification of the inositol residue during the construction of the GPI-anchor in the ER lumen is different among apicomplexans. Coccidia can both acylate (PIG-W) and deacylate (PGAP1) inositol, while P. falciparum and Babesia bovis can only acylate, and Cryptosporidium spp. have lost both capacities.

$\mathrm{N}$-linked glycans, oligosaccharides attached to the asparagine (Asn) residue in a tripeptide sequence of Asn$\mathrm{X}$-Ser/Thr (where $\mathrm{X}$ is any amino acid except Pro) of proteins, are very common in eukaryotes [20]. Based on the presence and absence of critical enzymes involved in the biosynthesis of $\mathrm{N}$-glycan precursors, we have predicted putative final $\mathrm{N}$-glycan precursor structures in different apicomplexans (Fig. 3b). Compared to T. gondii and Cryptosporidium spp., C. cayetanensis does not add any glucose onto the core structure of the $\mathrm{N}$-glycan precursor. In contrast, the enzymes that catalyze the addition of oligosaccharides onto the $\mathrm{N}$-acetylglucosamines (GlcNAc) during $\mathrm{N}$-glycan biosynthesis are absent in E. tenella, P. falciparum and B. bovis. During the trimming process, the glucosidase needed for removing the external glucose is absent in C. cayetanensis while another glucosidase involved in removing the remaining two glucoses is present.

Mucin type O-glycosylation is another common posttranslational modification of proteins especially those from the secretory organelles of apicomplexans [21]. The enzymes catalyzing the biosynthesis of O-glycans have not been characterized for apicomplexans, except 


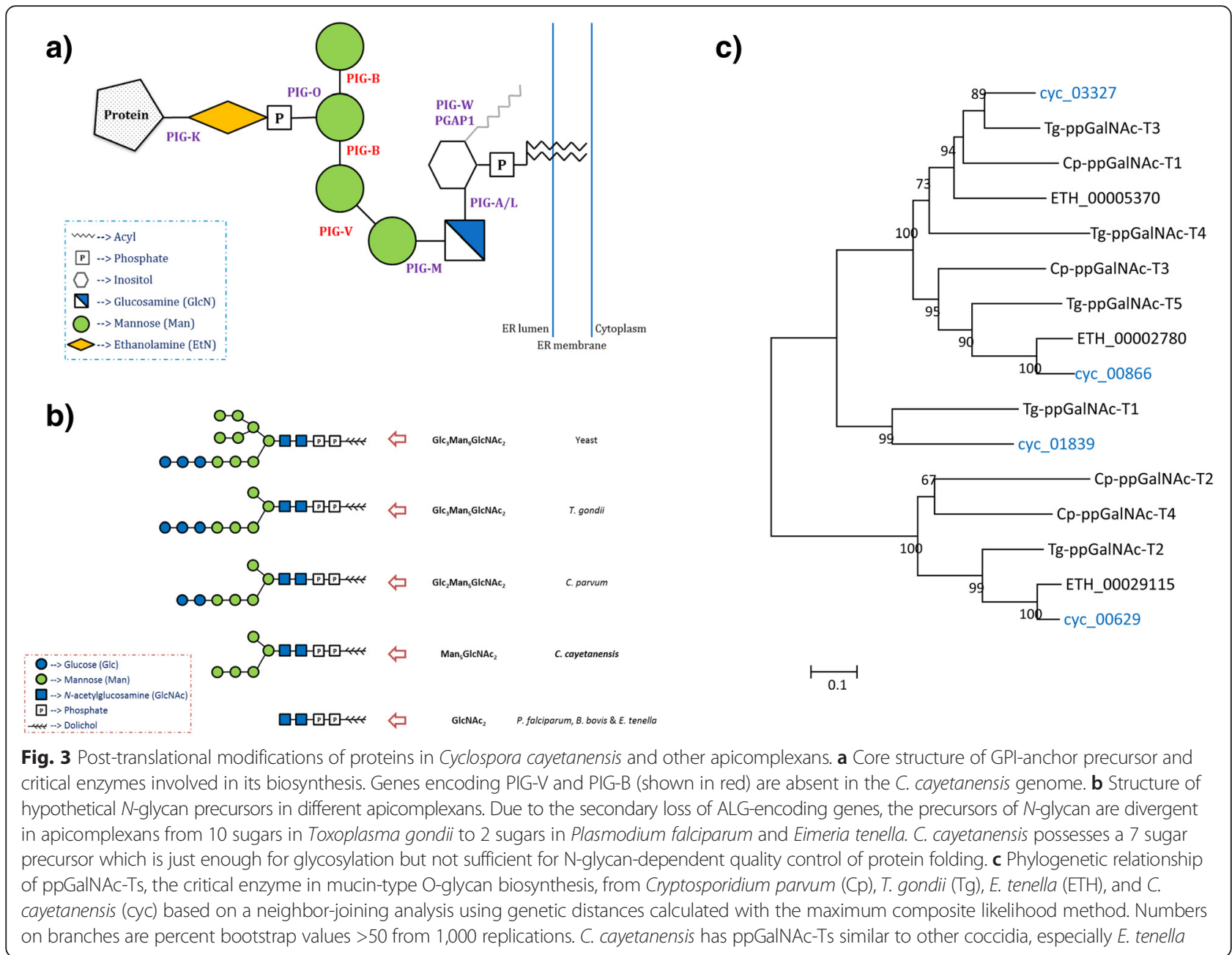

for the initial enzyme, UDP-GalNAc: polypeptide $N$ acetylgalactosaminyltransferase (ppGalNAc-T), which transfers GalNAc from UDP-GalNAc to the hydroxyl group of specific serine or threonine residues in proteins $[21,22]$. Four putative ppGalNAc-Ts from distinct families were identified in C. cayetanensis (Fig. 3c).

\section{Adhesins, surface antigens and glideosome}

By function, the super families of secreted proteins in the apical complex can be separated into three groups: i) adhesins involved in binding and interaction with host cells during the initial invasion, ii) secreted or membraneassociated peptidases involved in processing rhoptry and micronemal proteins of parasites and degrading proteins of the host, and iii) secreted signaling proteins such as protein phosphatases and kinases, which are injected across the plasma membranes into the host cell cytoplasm or nucleus, modulating host cell signaling pathways or immune responses to promote the survival of parasites [23]. Some of the major host cell invasion-related protein families were compared among common apicomplexans, which has shown some diversity in major surface antigens and protein kinases (Fig. 2d).

Based on the type of adhesins shared among parasites, C. cayetanensis and E. tenella probably have an adhesive system very similar to that of $T$. gondii (Additional file 7 : Table S4). Some major differences, however, were seen in the type of major surface antigens among coccidia. There are a large number of surface antigens called SRS proteins on the surface of $T$. gondii, approximately doubling the number in Neospora caninum, a close relative of $T$. gondii [24]. These highly expressed surface proteins are thought to be involved in the attachment of parasites to host cells and potentially to be responsible for the broad host range of T. gondii [25]. In E. tenella, the principal surface antigen genes (89 genes in three subfamilies) are arrayed in four gene clusters. Their products, TA4-type surface antigens containing signal peptides and GPIanchor sites, are thought to interact with host cell prior to invasion [11]. We did not find any cluster of genes encoding proteins with signal peptides and GPI-anchor sites in the $C$. cayetanensis genome. Only four putative TA4-type 
surface antigens, which are more similar to the subfamily SagA of Eimeria spp., were identified in C. cayetanensis and one of them has both a signal peptide and a GPIanchor. The cysteine-rich secretory protein family (CAP), which TA4 surface antigens probably derived from, was also detected in the C. cayetanensis genome. By paralog analysis using OrthoMCL, a large group comprised of 31 genes annotated as hypothetical proteins were found in the $C$. cayetanensis genome. Some $(n=11 / 31)$ have cytosol membrane-related or periplasmic substrate bindingrelated functions (Additional file 8: Table S5). One of these paralogs has some sequence similarity to erythrocyte membrane protein 1 (PfEMP1), which is involved in erythrocyte invasion by $P$. falciparum [26]. The length of these paralogous genes varies from $243 \mathrm{bp}$ to $3627 \mathrm{bp}$, compared with $\sim 700-800 \mathrm{bp}$ in the TA4 genes of $E$. tenella.

In T. gondii and P. falciparum, the power source of gliding and invasion comes from a motor complex consisting of myosin, gliding associated protein (GAP) and some other proteins [27]. The homologs of all of these proteins were found in C. cayetanensis and E. tenella suggesting that the motor structure may be conserved within all apicomplexans (Additional file 7: Table S4). After the initial attachment to host cells, $T$. gondii forms a moving junction, the AMA1-RON complex, to anchor the parasite to the host cell cytoskeleton [27, 28]. C. cayetanensis and E. tenella possess homologs for these proteins, suggesting that their host cell attachment system is similar to that in $T$. gondii.

\section{Secreted proteases and protein kinases}

Proteases and peptidases produced by the apical complex are thought to either modify other secreted apical complexrelated proteins that function in the extracellular environment or degrade host proteins after crossing the plasma membrane. One serine protease, subtilisin in $T$. gondii (TgSUB1), is required for the processing of microneme proteins, affecting the efficiency of adhesion of tachyzoites [29]. Although the ortholog of TgSUB1 was not found in $C$. cayetanensis and E. tenella, the ortholog of another rhoptry subtilisin-like protease with specificity similar to the ROP1 mutarase [30], TgSUB2, was found in these two parasites (Additional file 9: Table S6). Thus far, two cysteine endoproteases, cathepsins $\mathrm{B}(\mathrm{TgCPB})$ and $\mathrm{L}(\mathrm{TgCPL})$, and three cysteine exoproteases, cathepsins $\mathrm{C} 1$ to $\mathrm{C} 3$ ( $\mathrm{TgCPC} 1$, $\mathrm{TgCPC} 2$ and $\mathrm{TgCPC} 3$ ), have been characterized in T. gondii and are known to play essential roles in the growth and intracellular survival of parasites [31]. Except for CPC3, which is present in E. tenella, C. cayetanensis has four members of these two types of proteases. Even though the substrate for metalloproteinases, named toxolysins, is unclear, the presence of a rhoptry pro-domain cleavage site within toxolysin-1 (TLN1) suggests that toxolysins are probably protein maturases [32]. Rhomboid proteases (ROMs) are a family of intramembrane serine proteases in all kingdoms of life, and were shown to be responsible for the cleavage of secreted adhesive proteins in apicomplexans. Among them, TgROM4 functions as a micronemal protease and is essential for host cell invasion of $T$. gondii [33, 34], whereas TgROM2 and TgROM5 are thought to cleave the transmembrane domains of some MICs that are involved in gliding and invasion [35]. The homolog of TgROM3 was not identified in C. cayetanensis, whereas homologs of TgROM2 and TgROM6 were not identified in $E$. tenella (Additional file 9: Table S6).

Apicomplexans have the ability to modulate host cell metabolism, especially the signaling pathways to allow them to evade the host immune system. T. gondii possesses a special secretory protein phosphatase $2 \mathrm{C}$ (PP2Chn) secreted by the rhoptry and delivered into host cell nuclei during invasion [36]. There are no orthologs of PP2C-hn in C. cayetanensis and E. tenella (Additional file 10: Table S7). Some PP2C-like secretory phosphatases were identified in C. cayetanensis and E. tenella, but their numbers are smaller than seen in T. gondii. In addition, rhoptries also release a range of protein kinases (ROPK) to modulate host cell functions. The best known is TgROP18, which phosphorylates and inactivates host immunity-related GTPases [37, 38]. ROP5, ROP16 and ROP38 are also implicated in the modulation of host immune responses or signaling pathways. These ROPKs do not have any orthologs within $C$. cayetanensis and $E$. tenella. E. tenella has a smaller number of ROPKs and several E. tenella-specific groups of ROPKs [11]. We identified 13 putative ROPK-encoding genes in the C. cayetanensis genome, significantly smaller than the number in the E. tenella genome but similar to that in E. falciformis [39] (Table 4). The putative ROPKs of $C$. cayetanensis are ROP21/27/35-like and E. tenella-specific ROPKs (Fig. 4a). Among them, ROP21/27, ROP35 and ROPK-Eten1 subfamilies have conserved catalytic residues of ROPKs [40] (Fig. 4b), suggesting that these coccidia likely have some capacity to modify host signaling pathways. Overall, the number of known secretory ROPKs in $C$. cayetanensis is significantly reduced, and two of them, ROPK-Eten 4 and ROPK-Eten5, appear to be orthologs of E. tenella ROPKs.

In $T$. gondii, there are two potent nucleoside triphosphate hydrolases, NTPase I and NTPase II, which are localized in dense granules and secreted into the $\mathrm{PV}$, affecting host signaling pathways during invasion [23]. Both of them are absent in C. cayetanensis and $E$. tenella. Protease inhibitors in T. gondii, TgPI-1 and TgPI-2, are dense granule proteins secreted into the PV to potentially inhibit trypsin, chymotrypsin, neutrophil and pancreatic elastases, protecting the parasite from host immune responses [41]. The lack of these catalytic proteins with functional domains, such 
Table 4 Predicted rhoptry protein kinases (ROPKs) in Cyclospora cayetanensis using HMM profiles search and their orthologs in other coccidia

\begin{tabular}{|c|c|c|c|c|c|c|c|}
\hline Gene ID & Best hit HMM family & E-value & Score & General PK score & E. tenella & E. falciformis & T. gondii \\
\hline cyc_02428 & ROP21/27 & $1.4 \mathrm{E}-104$ & 348.6 & 94.4 & ETH_00014495 & EfaB_PLUS_7742.g778 & TGME49_263220 \\
\hline cyc_03750 & ROP21/27 & $3.1 \mathrm{E}-100$ & 334.4 & 82.9 & & EfaB_PLUS_47595.92679 & TGME49_313330 \\
\hline cyc_04230 & ROP35 & $1.6 \mathrm{E}-39$ & 134.9 & 40.3 & ETH_00005905 & EfaB_MINUS_42996.g2710 & \\
\hline cyc_03158 & ROP35 & $4.3 E-83$ & 277.5 & 89.3 & ETH_00026495 & EfaB_PLUS_8664.g829 & TGME49_304740 \\
\hline cyc_00988 & ROPK-Eten 1 & 3.0E-108 & 361.3 & 75.0 & ETH_00027705 & EfaB_PLUS_15899.g1411 & \\
\hline cyc_00989 & ROPK-Eten 1 & $2.6 \mathrm{E}-77$ & 259.6 & 79.2 & ETH_00027695 & & \\
\hline cyc_03944 & ROPK-Eten 1 & 4.0E-29 & 100.8 & 57.2 & ETH_00027700 & & \\
\hline \multirow[t]{2}{*}{ cyc_05579 } & ROPK-Eten2a & $3.9 \mathrm{E}-60$ & 202.5 & 78.4 & ETH_00028765 & & \\
\hline & ROPK-Eten2b & & & & ETH_00028855 & & \\
\hline \multirow[t]{7}{*}{ cyc_08168 } & ROPK-Eten3 & $1.1 \mathrm{E}-35$ & 122.2 & 40.4 & ETH_00020585 & & \\
\hline & ROPK-Eten3 & & & & ETH_00020615 ETH_00020590 & & \\
\hline & & & & & ETH_00020610 ETH_00005840 & & \\
\hline & & & & & ETH_00021185 ETH_00020620 & & \\
\hline & ROPK-Eten4 & & & & ETH_00000075 ETH_00000080 & & \\
\hline & ROPK-Eten5 & & & & ETH_00005415 ETH_00005400 & & \\
\hline & & & & & ETH_00005405 ETH_00005410 & & \\
\hline cyc_02713 & ROPK-Eten6 & $1.6 \mathrm{E}-66$ & 223.3 & 64.0 & ETH_00002510 & EfaB_MINUS_32658.g2475 & \\
\hline cyc_05580 & ROPK-Unique & $1.3 \mathrm{E}-71$ & 240.3 & 78.2 & ETH_00028835 & EfaB_MINUS_17096.g1521 & \\
\hline cyc_04110 & ROPK-Unique & $4.1 \mathrm{E}-56$ & 189.4 & 28.0 & ETH_00013325 & EfaB_PLUS_24117.g1969 & \\
\hline \multirow[t]{2}{*}{ cyc_07646 } & ROPK-Unique & $1.1 \mathrm{E}-48$ & 165.0 & 41.4 & ETH_00005170 & EfaB_PLUS_33184.92393 & \\
\hline & ROPK-Unique & & & & ETH_00005335 & & \\
\hline
\end{tabular}

as Kazal in TgPI proteins, may be partially responsible for the strict tissue tropism in $C$. cayetanensis and $E$. tenella. There is also a large group of secretory proteins stored in dense granules called GRAs in T. gondii, which have no identifiable Pfam domains but are essential for invasion and egress. One of them, GRA15, like some rhoptry proteins is delivered across the PV membrane to modulate host cell signal pathways. Two others, GRA16 and GRA24, have been demonstrated to target the host cell nucleus, affecting host gene expression [9]. Except for GRA9/10/11/12, there are no homologs of these proteins in C. cayetanensis and E. tenella.

\section{Transcription factors}

Apicomplexans have a major transcription factor family called the apicomplexan AP2 family of proteins (ApiAP2), with some similarities to the plant AP2 $[42,43]$. In $T$. gondii, TgAP2s regulate stage-specific expression of genes. At least 35 ApiAP2 domain-containing proteins are encoded by $C$. cayetanensis. This is less than the 44 ApiAP2 proteins in T. gondii, but more than the 27 in E. tenella.

\section{Discussion}

Comparative genomic analysis indicates that $C$. cayetanensis shares some of the genomic features and metabolic capabilities of coccidia such as $T$. gondii and $E$. tenella. Compared with the metabolism in T. gondii, $C$. cayetanensis and E. tenella primarily lack de novo biosynthesis of certain amino acids and the ability to salvage amino acids directly from the host is significantly reduced. Differences in the degradation and hydroxylation pathways of some amino acids were also observed among the coccidian parasites examined. It appears amino acid metabolism evolves more rapidly in coccidia than other metabolic pathways. It is possible the lack of these amino acid metabolic pathways has reduced the target tissue range in $C$. cayetanensis and E. tenella. The only unique metabolic pathway present in $C$. cayetanensis and E. tenella but absent in T. gondii is the synthesis of mannitol from fructose catalyzed by a single enzyme. The fungi-like mannitol cycle metabolism (fructosemannitol-mannitol phosphate-fructose phosphatefructose) was known to be present in E. tenella [44]. Mannitol is accumulated as an energy reserve during oocyst formation in the host and utilized for sporulation 


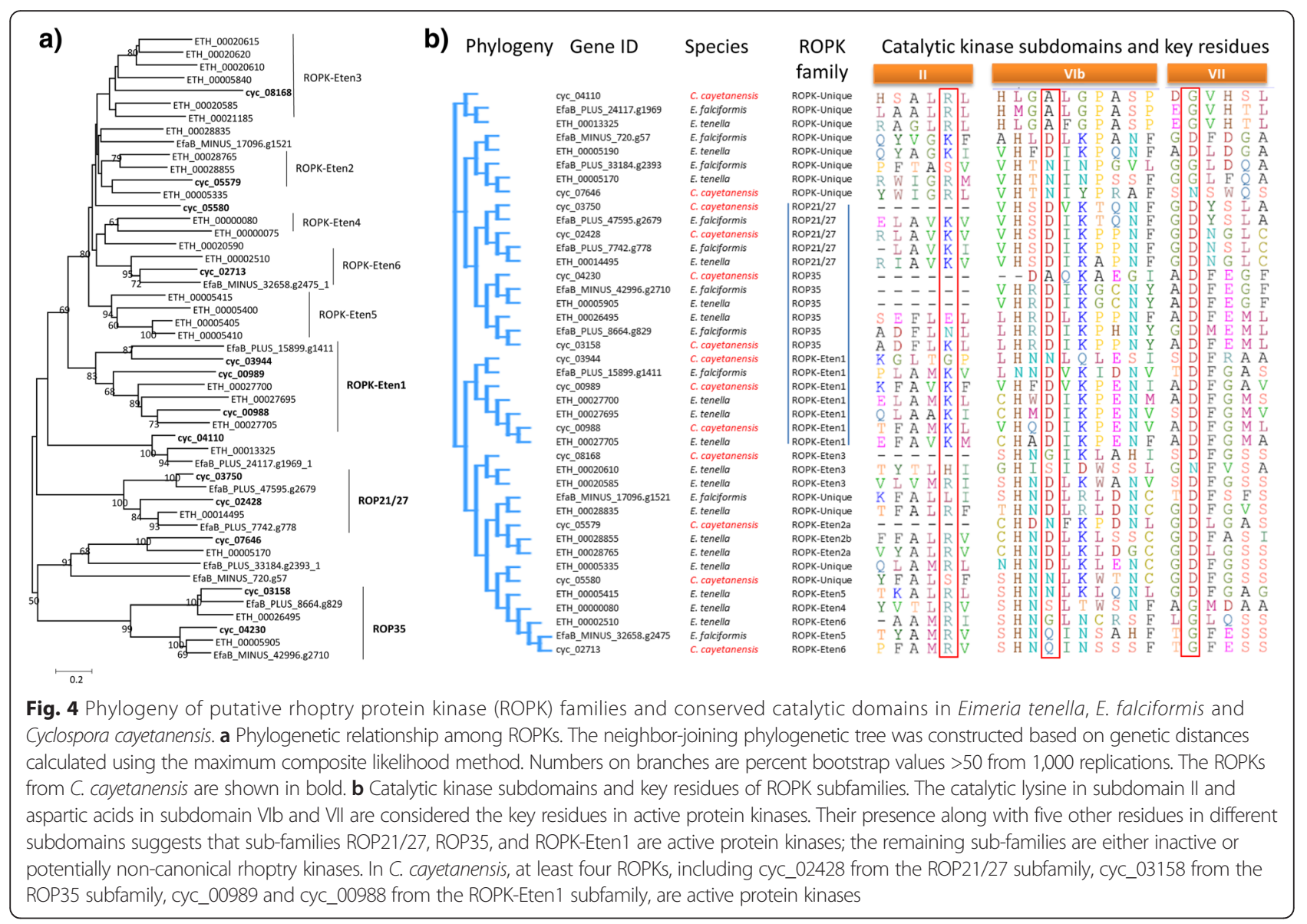

outside of the host [45]. It is probably vital to the sporulation and survival of $C$. cayetanensis oocysts. A clear difference between $C$. cayetanensis and E. tenella is the ability of the former to degrade propionyl-CoA to produce pyruvate and succinate, probably as supplements for the TCA cycle, suggesting that $C$. cayetanensis probably has more carbon sources for mitochondrial metabolic activities than $E$. tenella.

The mechanisms involved in post-translational modifications of proteins may be different between C. cayetanensis and Eimeria spp. Because of the secondary loss of ALG enzymes, the length of $\mathrm{N}$-glycan precursors is divergent among apicomplexans, from 10 sugars in $T$. gondii to 2 in E. tenella and none in Theileria spp. [46]. The Nglycan precursor in C. cayetanensis possesses 7 sugars, $\mathrm{Man}_{5} \mathrm{GlcNAc}_{2}$. Similarly, a paucity of enzymes involved in the biosynthesis of GPI-anchor was detected in $C$. cayetanensis and E. tenella. Both $C$. cayetanensis and $E$. tenella lack mannosyltransferases PIG-V and PIG-B. In addition, phosphomannomutase and dolichol-phosphatemannose (Dol-P-Man) synthase, the enzymes involved in generation of Dol-P-Man, which is the substrate for Nglycan and GPI-anchor synthesis, are absent in Eimeria spp. [39] but are present in C. cayetanensis. Thus, there are substantial differences in both GPI anchor biosynthesis and $\mathrm{N}$-glycosylation between $C$. cayetanensis and Eimeria spp.

The similar repertoire of host cell invasion-related proteins possessed by all coccidian parasites suggests that $C$. cayetanensis has host cell receptors and invasion process similar to that of $T$. gondii and E. tenella: gliding powered by an actin motor before invasion, interactions with the host cell through surface antigens, forming a moving junction with a series of secreted proteins to initiate invasion, forming a PV structure inside the host cell, and secreting various protein kinases, protein phosphatases, and other catalytic proteins to modify the host metabolic pathways for the evasion of host immune responses. The amplification and diversification of surface and secreted proteins are probable determinants for distinct transmission, host range and pathogenicity of various coccidia [47]. Compared with T. gondii, the dramatic reduction in protein kinases and phosphatases suggests that $C$. cayetanensis and E. tenella have only limited capacity to regulate host cell signaling pathways and gene expression, or use a divergent system to do so. These genomic characteristics of E. tenella and C. cayetanensis are probably responsible for their exclusive enteric life 
cycle. Between $C$. cayetanensis and $E$. tenella, the former has a further reduction in the number and type of ROPKs.

Surface antigens SRS (SAG1-related sequences) are involved in initial interaction with host cells in $T$. gondii invasion [48]. N. caninum possesses the same type of SRS proteins seen in T. gondii, but has a significant increase in their number [24]. Neither $C$. cayetanensis nor E. tenella has this family of surface proteins. In contrast, Eimeria spp. have the unique TA4-type surface antigens and show divergence in their compositions among species [11]. In the $C$. cayetanensis genome, we detected several TA4-type surface antigen coding genes in different genomic regions. Thus, surface antigens are probably the most rapidly evolved proteins in coccidia and are likely determinants for host specificity. We assume that $C$. cayetanensis possesses its own unique surface antigens. The paralogous genes we identified in this study encode mostly hypothetical proteins, one of which has sequence homology to the PfEMP1 of $P$. falciparum. They probably represent the surface antigens of $C$. cayetanensis, as many of these proteins are predicted to have membrane-related or periplasmic substrate binding-related functions. Further studies on the expression, localization and neutralization ability of these proteins are needed to confirm their surface antigen nature in C. cayetanensis.

\section{Conclusions}

Through whole genome sequencing and comparative genomic analysis, we have shown that $C$. cayetanensis probably possesses a classical coccidian metabolism and has a host cell invasion system very similar to Eimeria spp. and T. gondii. The amino acid metabolism and post-translation modifications of proteins are probably the most rapidly evolved metabolic pathways among coccidia. Compared with the heteroxenous $T$. gondii, the monoxenous C. cayetanensis and Eimeria spp. appear to have very limited abilities or use different mechanisms to modulate host nuclear activities and signaling pathways during invasion. The dominant surface antigens seen in other coccidia are not present or are significantly reduced in number in $C$. cayetanensis and the presence of divergent surface proteins among coccidia suggests that these proteins are likely determinants of host specificity. These observations, however, are based on results of comparative genomic analyses and need to be validated by functional studies. Overall, the availability of whole genome sequence data has significantly improved our understanding of the biology of C. cayetanensis and may facilitate the development of molecular diagnostic tools for traceback studies of foodborne cyclosporiasis outbreaks.

\section{Methods}

\section{Sample collection and DNA preparation}

The C. cayetanensis specimen sequenced in this study was collected in July 2011 from a patient with severe diarrhea in Kaifeng, Henan, China, where cyclosporiasis is endemic and $C$. cayetanensis isolates were characterized morphologically and by sequence analysis of the SSU rRNA gene [49]. It was diagnosed in this study through acid-fast microscopy and confirmed as $C$. cayetanensis by ultraviolet epifluorescence microscopy and PCR analysis of a $~ 680$ bp fragment of the SSU rRNA gene [10]. DNA sequences obtained from three PCR products were identical to each other and had only an A to G substitution at nucleotide 72 of the GenBank reference sequence AF111183. C. cayetanensis oocysts were purified from the specimen using sucrose and cesium chloride gradients [50] and further purified twice by flow cytometry sorting on a FACSAria III (BD Biosciences, San Jose, CA). A gate on forward and side scatter profiles, a gate on autofluorescence, and detectors and filters appropriate for propidium iodide (PI) and fluorescein isothiocyanate were used in sorting. The oocysts in suspension were stained with 1.5 micrograms $/ \mathrm{ml}$ of PI and a $488 \mathrm{~nm}$ laser was used for excitation. Total genomic DNA was extracted from $6 \times 10^{6}$ oocysts using a QIAamp ${ }^{\circ}$ NA Mini Kit (Qiagen Sciences, Maryland, 20874, USA), after the oocysts were subjected to five freeze-thaw cycles and overnight digestion with proteinase K. About 100 ng of extracted DNA was amplified using REPLI-g Midi Kit (Qiagen GmbH, Hilden, Germany) according to the manufacturer-recommended procedure.

\section{Library construction, sequencing, and assembly}

The C. cayetanensis isolate was sequenced on a Roche 454 GS-FLX Titanium System (Roche, Branford, CT) using the standard Roche library protocol, and on an Illumina Genome Analyzer IIx and a Hiseq 2500 (Illumina, San Diego, CA) using the Illumina TruSeq (v3) library proto$\mathrm{col}$. For Roche 454 sequencing, sequence reads of approximately $400 \mathrm{bp}$ were generated in one run and $450 \mathrm{bp}$ in another, whereas in Illumina sequencing, $100 \times 100 \mathrm{bp}$ paired-end reads were generated. The raw sequencing reads from the two platforms were combined, and reads of quality score below 30 were trimmed using CLC Genomics Workbench 7.03 (http://www.clcbio.com/products/ clc-genomics-workbench). They were assembled into contigs using the default parameters.

\section{Structural analysis of genome}

The BLASTN [51] program was used to analyze the assembled contigs with data in GenBank. Contigs from contaminating organisms were removed using a threshold e-value of $1 \mathrm{e}-10$ and manual inspections of the sequence coverages. BUSCO [52] was used to search the 429 core eukaryotic orthologs within genomes of $T$. gondii, E. tenella and $C$. 
cayetanensis and assess the completeness of the genome sequencing. Simple tandem repeat and low complexity sequences in the C. cayetanensis genome were identified using RepeatMasker version 4.0.3 (http://repeatmasker.org/), whereas LTR-retrotransposons were identified using LTRharvest [53]. Circos [54] was used to present the alternating patterns of repeat-rich and repeat-poor sequences in long contigs.

All predicted LTR-retrotransposons were extracted and translated into amino acid sequences. HMMER (http:// hmmer.janelia.org/) was used to search chromodomain (PF00385) and reverse transcriptase (PF00078) motifs in these sequences using the HMM model from Pfam [55] (http://pfam.xfam.org/). A cluster analysis of all LTRretrotransposons was conducted based on nucleotide sequence identities. The longest LTR-retrotransposon from the biggest group was used for phylogenetic analysis. The chromovirus-type LTR sequence of E. tenella was randomly chosen and other LTR-retrotransposons were retrieved from NCBI GenBank. ClustalX v2 [56] was used in the preparation of a sequence alignment of LTR retrotransposons and MEGA v6 [57] was used in the construction of a neighbor-joining tree with the maximum composite likelihood mode for distance calculation and 1000 replications for bootstrapping.

Two command line software packages, tRNAscan-SE v1.3.1 [58] and ARAGORN v1.2.36 [59], were used to identify tRNA genes in the $C$. cayetanensis genome. Both of them were executed using the default settings and the general tRNA model or standard genetic codon, with the final results combined. Ribosomal RNA genes were identified using RNAmmer v1.2 [60]. Other genomic features were identified using in-house scripts.

\section{Gene prediction and functional annotation}

Protein-encoding genes in the $C$. cayetanensis genome were predicted using a pipeline of three software packages, including AUGUSTUS v2.7 [61], SNAP [62], and GeneMark-ES [63]. AUGUSTUS and SNAP were trained with the gene-model of E. tenella (ToxoDB release-11.0), while GeneMark-ES is a selftraining gene predictor. After examination of outcomes of gene predictions (data not shown), we kept all genes predicted by AUGUSTUS, because they fit well into the gene model. New genes predicted by both SNAP and GeneMark-ES were combined with the results of AUGUSTUS as the final protein-coding gene set of $C$. cayetanensis. SignalP v4.1 [64] and TMHMM v2.0 [65] with default settings were used to identify signal peptides and transmembrane domains within the predicted proteins, respectively. Proteins targeting the apicoplast were predicted using ApicoAP
[66]. GPI anchor attachment signals were identified using the GPI-SOM webserver [67].

\section{Metabolism and invasion-related protein analysis}

A BLASTP [51] search of the GenBank NR database and a webserver KAAS [68] were used to map the predicted proteins to specific cellular metabolic pathways. We consider the parasite to possess a certain pathway if it has the gene encoding the essential enzymes for it. The comparison of metabolism among apicomplexans was based on these analytic results and data from public databases LAMP (Library of Apicomplexan Metabolic Pathways, release-2) [12] and EuPathDB (http://eupathdb.org/eupathdb/).

Orthologs of other apicomplexans in the predicted proteome of $C$. cayetanensis were identified by using OrthoMCL [69]. Groups of paralogs within the genome were also identified by inspection of the results. The potential functions of the largest group of paralogs were identified through BLASTP analysis against the GenBank database. The identification of apical complex proteins and protein domains were conducted using the webserver Pfam [55]. Venn diagrams of protein domains shared by five apicomplexans were drawn by using the Venny tool (http://bioinfogp.cnb.csic.es/tools/venny/index.html). The phylogenetic relationship between $C$. cayetanensis and common apicomplexans was assessed by neighbor-joining analysis of an alignment of concatenated protein sequences of 100 orthologs, as described by Woo [70]. Gblocks [71] was used to remove the highly divergent regions before the construction of the phylogenetic tree.

A comparison of transporter proteins was conducted based on the Pfam search results. The database for coccidia-specific rhoptry kinases and pseudokinases HMM profiles [40], which classifies ROPKs from the genomes of T. gondii, N. caninum, E. tenella, and other apicomplexans into 42 distinct subfamilies, was used in the prediction and analysis of ROPKs in C. cayetanensis with the best hit score threshold set at 100. All putative ROPKs sequences identified in E. tenella, E. falciformis, and C. cayetanensis were extracted and analyzed with the neighbor-joining method described above.

\section{Ethics approval and consent to participate}

The genome sequencing was done on a delinked residual diagnostic specimen. The work was covered by Human Subjects Protocol No. 990115 "Use of residual human specimens for the determination of frequency of genotypes or sub-types of pathogenic parasites," which was reviewed and approved by the Institutional Review Board of the Centers for Disease Control and Prevention (CDC). No personal identifier was associated with the specimen at the time of its submission for diagnostic service at CDC. 


\section{Availability of data and materials}

The datasets supporting the conclusion of this article, including all Sequence Read Archive (SRA) data (SRX665300 and SRX681889), assembled contigs (ASM76915v1), and annotations (JROU00000000) are available in the NCBI BioProject under the accession No. PRJNA256967. The phylogenetic data supporting the conclusions of this article are available in the TreeBase (http://purl.org/phylo/treebase/phylows/study/TB2:S19120).

\section{Additional files}

Additional file 1: Figure S1. De novo assembly of Cyclospora cayetanensis. A total of 4,811 contigs with an overall length of $46,816,962 \mathrm{bp}$, mean length of 9,713 bp, and N50 contig length of 55,741 bp, were generated in the de novo assembly of sequences. (DOCX $71 \mathrm{~kb}$ )

Additional file 2: Table S1. Summary of Cyclospora cayetanensis genome. (DOCX $14 \mathrm{~kb}$ )

Additional file 3: Table S2. Assessment of the completeness of sequenced Toxoplasma gondii, Eimeria tenella and Cyclospora cayetanensis genomes based on core eukaryotic protein-encoding genes search using BUSCO. (DOCX 14 kb)

Additional file 4: Figure S2. Predicted LTR-retrotransposons in Cyclospora cayetanensis. A total of 87 LTR-retrotransposons were detected in C. cayetanensis. The $x$-axis represents the length of LTR-retrotransposons, and $y$-axis represents the average lengths of upstream and downstream LTRS of each retrotransposon. The darkness represents the sequence similarities between the upstream and downstream LTRs of each retrotransposon. (DOCX $83 \mathrm{~kb}$ )

Additional file 5: Figure S3. Evolutionary relationship of LTRretrotransposons based on neighbor-joining analysis using genetic distances calculated with the maximum composite likelihood method. The LTR-retrotransposon in Eimeria tenella is placed within the clade formed by chromoviruses which are widely present in eukaryotic genomes. The LTR-retrotransposon of Cyclospora cayetanensis is clearly out of the clade. Numbers on branches are percent bootstrap values $>50$ from 1,000 replications. (DOCX $58 \mathrm{~kb}$ )

Additional file 6: Table S3. Comparison of essential cellular metabolic pathways among some common apicomplexan parasites. (XLSX $70 \mathrm{~kb}$ )

Additional file 7: Table S4. Comparison of host cell invasion-related adhesins among Toxoplasma gondii, Eimeria tenella, and Cyclospora cayetanensis. (DOCX $29 \mathrm{~kb}$ )

Additional file 8: Table S5. Members of a major group of paralogs detected in the Cyclospora cayetanensis genome and results of BLASTP against the GenBank protein database. (XLSX 33 kb)

Additional file 9: Table S6. Comparison of host cell invasion-related peptidases and proteases among Toxoplasma gondii, Eimeria tenella, and Cyclospora cayetanensis. (DOCX $24 \mathrm{~kb}$ )

Additional file 10: Table S7. Comparison of host cell invasion-related protein phosphatases, kinases, and other signaling related proteins among Toxoplasma gondii, Eimeria tenella, and Cyclospora cayetanensis. (DOCX $25 \mathrm{~kb})$

\section{Abbreviations \\ AMA1: apical membrane antigen 1; CoA: coenzyme A; EMP1: erythrocyte membrane protein 1; GPI: glycosylphosphatidylinositol; GRA: dense granule protein; ICDH: isocitrate dehydrogenase; LA: lipoic acid; LTR: long terminal repeat; MIC: microneme protein; PEP: phosphoenolpyruvate; PLP: pyridoxal 5-phosphate; PRPP: phosphoribosyl pyrophosphate; PV: parasitophorous vacuole; RON: rhoptry neck protein; ROP: rhoptry protein; ROPK: rhoptry protein kinases; SAG: surface antigen; SRS: SAG1-related sequences; STR: short tandem repeat; TCA: tricarboxylic acid; THF: tetrahydrofolate.}

\section{Competing interests}

The authors declare that they have no competing interests.

\section{Authors' contributions}

YF and LX conceived and designed the experiments; SL, LW, HZ, ZX, DMR, $N L, M A F, K T, M J A, D M M$ and LZ performed the experiments; $S L, H Z$, YF, and $L X$ analyzed the data; SL, YF and LX wrote the paper. All authors read and approved the final manuscript.

\section{Acknowledgements}

The findings and conclusions in this report are those of the authors and do not necessarily represent the views of the US Centers for Disease Control and Prevention.

\section{Funding}

This work was supported by the National Natural Science Foundation of China (31425025 and 31229005), Open Funding Project of the State Key Laboratory of Veterinary Etiological Biology, Lanzhou, China (SKLVEB2014KFKT008), and the US Centers for Disease Control and Prevention.

\section{Author details}

${ }^{1}$ State Key Laboratory of Bioreactor Engineering, School of Resources and Environmental Engineering, East China University of Science and Technology, Shanghai 200237, China. ${ }^{2}$ Division of Foodborne, Waterborne, and Environmental Diseases, Centers for Disease Control and Prevention, Atlanta, GA 30333, USA. ${ }^{3}$ Shanghai-Ministry of Science and Technology Key Laboratory of Health and Disease Genomics, Chinese National Human Genome Center at Shanghai, 250 Bibo Road, Shanghai 201203, China.

${ }^{4}$ Division of Scientific Resources, Centers for Disease Control and Prevention, Atlanta, GA 30333, USA. ${ }^{5}$ College of Animal Science and Veterinary Medicine, Henan Agricultural University, Zhengzhou 450002, China.

Received: 10 December 2015 Accepted: 20 April 2016

Published online: 30 April 2016

\section{References}

1. Ortega YR, Sanchez R. Update on Cyclospora cayetanensis, a food-borne and waterborne parasite. Clin Microbiol Rev. 2010;23(1):218-34.

2. Chacin-Bonilla L. Epidemiology of Cyclospora cayetanensis: A review focusing in endemic areas. Acta Trop. 2010:115(3):181-93.

3. Abanyie F, Harvey RR, Harris JR, Wiegand RE, Gaul L, Desvignes-Kendrick M, Irvin K, Williams I, Hall RL, Herwaldt B, et al. 2013 multistate outbreaks of Cyclospora cayetanensis infections associated with fresh produce: focus on the Texas investigations. Epidemiol Infect. 2015;143:1-8.

4. Tenter AM, Heckeroth AR, Weiss LM. Toxoplasma gondii: from animals to humans. Int J Parasitol. 2000;30(12-13):1217-58.

5. Butcher BA, Reese ML, Boothroyd JC, Denkers EY. Interactions between Toxoplasma effectors and host immune responses. In: Toxoplasma gondii: The Model Apicomplexan - Perspectives and Methods: Second Edition. Boston: Elsevier Ltd.; 2014: 505-519.

6. Paing MM, Tolia NH. Multimeric assembly of host-pathogen adhesion complexes involved in apicomplexan invasion. PLoS Pathog. 2014;10(6):e1004120.

7. Lim DC, Cooke BM, Doerig C, Saeij JP. Toxoplasma and Plasmodium protein kinases: roles in invasion and host cell remodelling. Int J Parasitol. 2012;42(1):21-32.

8. Hunter CA, Sibley LD. Modulation of innate immunity by Toxoplasma gondii virulence effectors. Nat Rev Microbiol. 2012;10(11):766-78.

9. Mercier C, Cesbron-Delauw MF. Toxoplasma secretory granules: one population or more? Trends Parasitol. 2015;31(2):60-71.

10. Tang K, Guo Y, Zhang L, Rowe LA, Roellig DM, Frace MA, Li N, Liu S, Feng Y, Xiao L. Genetic similarities between Cyclospora cayetanensis and cecuminfecting avian Eimeria spp. in apicoplast and mitochondrial genomes. Parasite Vector. 2015;8:358.

11. Reid AJ, Blake DP, Ansari HR, Billington K, Browne HP, Bryant J, Dunn M, Hung SS, Kawahara F, Miranda-Saavedra D, et al. Genomic analysis of the causative agents of coccidiosis in domestic chickens. Genome Res. 2014;24(10):1676-85.

12. Shanmugasundram A, Gonzalez-Galarza FF, Wastling JM, Vasieva O, Jones AR. Library of apicomplexan metabolic pathways: a manually curated database for metabolic pathways of apicomplexan parasites. Nucleic Acids Res. 2013; 41 (Database issue):D706-713.

13. Coppin A, Varre JS, Lienard L, Dauvillee D, Guerardel Y, Soyer-Gobillard MO, Buleon A, Ball S, Tomavo S. Evolution of plant-like crystalline storage 
polysaccharide in the protozoan parasite Toxoplasma gondii argues for a red alga ancestry. J Mol Evol. 2005;60(2):257-67.

14. Seeber F, Soldati-Favre D. Metabolic pathways in the apicoplast of apicomplexa. Int Rev Cell Mol Biol. 2010;281:161-228.

15. Pino P, Foth BJ, Kwok LY, Sheiner L, Schepers R, Soldati T, Soldati-Favre D. Dual targeting of antioxidant and metabolic enzymes to the mitochondrion and the apicoplast of Toxoplasma gondii. PLoS Pathog. 2007;3(8):e115.

16. Fleige T, Fischer K, Ferguson DJ, Gross U, Bohne W. Carbohydrate metabolism in the Toxoplasma gondii apicoplast: localization of three glycolytic isoenzymes, the single pyruvate dehydrogenase complex, and a plastid phosphate translocator. Eukaryot Cell. 2007;6(6):984-96.

17. Donaldson TM, Cassera MB, Ho MC, Zhan C, Merino EF, Evans GB, Tyler PC, Almo SC, Schramm VL, Kim K. Inhibition and structure of Toxoplasma gondii purine nucleoside phosphorylase. Eukaryot Cell. 2014;13(5):572-9.

18. Cassera MB, Hazleton KZ, Riegelhaupt PM, Merino EF, Luo M, Akabas MH, Schramm VL. Erythrocytic adenosine monophosphate as an alternative purine source in Plasmodium falciparum. J Biol Chem. 2008;283(47):32889-99.

19. Crawford MJ, Thomsen-Zieger N, Ray M, Schachtner J, Roos DS, Seeber F. Toxoplasma gondii scavenges host-derived lipoic acid despite its de novo synthesis in the apicoplast. EMBO J. 2006;25(13):3214-22.

20. Stanley P, Schachter H, Taniguchi N: N-Glycans. In: Essentials of Glycobiology. Edited by Varki A, Cummings RD, Esko JD, Freeze HH, Stanley P, Bertozzi CR, Hart GW, Etzler ME, 2nd edn. Boston: Cold Spring Harbor (NY); 2009.

21. Stwora-Wojczyk MM, Kissinger JC, Spitalnik SL, Wojczyk BS. Oglycosylation in Toxoplasma gondii: identification and analysis of a family of UDP-GalNAc:polypeptide N-acetylgalactosaminyltransferases. Int J Parasitol. 2004;34(3):309-22

22. Bhat N, Wojczyk BS, DeCicco M, Castrodad C, Spitalnik SL, Ward HD. Identification of a family of four UDP-polypeptide $\mathrm{N}$-acetylgalactosaminyl transferases in Cryptosporidium species. Mol Biochem Parasitol. 2013;191(1):24-7.

23. Anantharaman V, Iyer LM, Balaji S, Aravind L. Adhesion molecules and other secreted host-interaction determinants in Apicomplexa: insights from comparative genomics. Int Rev Cytol. 2007;262:1-74.

24. Reid AJ, Vermont SJ, Cotton JA, Harris D, Hill-Cawthorne GA, Konen-Waisman S, Latham SM, Mourier T, Norton R, Quail MA, et al. Comparative genomics of the apicomplexan parasites Toxoplasma gondii and Neospora caninum: Coccidia differing in host range and transmission strategy. PLoS Pathog. 2012:8(3):e1002567.

25. Boothroyd JC. Expansion of host range as a driving force in the evolution of Toxoplasma. Mem Inst Oswaldo Cruz. 2009:104(2):179-84.

26. Hviid L, Jensen AT. PfEMP1 - A parasite protein family of key importance in Plasmodium falciparum malaria immunity and pathogenesis. Adv Parasitol. 2015:88:51-84.

27. Bargieri D, Lagal V, Andenmatten N, Tardieux I, Meissner M, Menard R. Host cell invasion by apicomplexan parasites: the junction conundrum. PLoS Pathog. 2014;10(9):e1004273.

28. Takemae H, Sugi T, Kobayashi K, Gong H, Ishiwa A, Recuenco FC, Murakoshi $F$, Iwanaga $T$, Inomata A, Horimoto $T$, et al. Characterization of the interaction between Toxoplasma gondii rhoptry neck protein 4 and host cellular beta-tubulin. Sci Rep. 2013;3:3199.

29. Lagal V, Binder EM, Huynh MH, Kafsack BF, Harris PK, Diez R, Chen D, Cole RN, Carruthers VB, Kim K. Toxoplasma gondii protease TgSUB1 is required for cell surface processing of micronemal adhesive complexes and efficient adhesion of tachyzoites. Cell Microbiol. 2010;12(12):1792-808.

30. Miller SA, Thathy V, Ajioka JW, Blackman MJ, Kim K. TgSUB2 is a Toxoplasma gondii rhoptry organelle processing proteinase. Mol Microbiol. 2003;49(4):883-94.

31. Que X, Engel JC, Ferguson D, Wunderlich A, Tomavo S, Reed SL. Cathepsin Cs are key for the intracellular survival of the protozoan parasite, Toxoplasma gondii. J Biol Chem. 2007:282(7):4994-5003.

32. Hajagos BE, Turetzky JM, Peng ED, Cheng SJ, Ryan CM, Souda P, Whitelegge JP, Lebrun M, Dubremetz JF, Bradley PJ. Molecular dissection of novel trafficking and processing of the Toxoplasma gondii rhoptry metalloprotease toxolysin-1. Traffic. 2012;13(2):292-304.

33. Rugarabamu G, Marq JB, Guerin A, Lebrun M, Soldati-Favre D. Distinct contribution of Toxoplasma gondii rhomboid proteases 4 and 5 to micronemal protein protease 1 activity during invasion. Mol Microbiol. 2015;97(2):244-62.

34. Shen B, Buguliskis JS, Lee TD, Sibley LD. Functional analysis of rhomboid proteases during Toxoplasma invasion. MBio. 2014;5(5):e01795-01714.

35. Santos JM, Graindorge A, Soldati-Favre D. New insights into parasite rhomboid proteases. Mol Biochem Parasitol. 2012;182(1-2):27-36.
36. Gilbert LA, Ravindran S, Turetzky JM, Boothroyd JC, Bradley PJ. Toxoplasma gondii targets a protein phosphatase $2 \mathrm{C}$ to the nuclei of infected host cells. Eukaryot Cell. 2007;6(1):73-83.

37. Steinfeldt T, Konen-Waisman S, Tong L, Pawlowski N, Lamkemeyer T, Sibley LD, Hunn JP, Howard JC. Phosphorylation of mouse immunity-related GTPase (IRG) resistance proteins is an evasion strategy for virulent Toxoplasma gondii. PLoS Biol. 2010;8(12):e1000576.

38. Fentress SJ, Behnke MS, Dunay IR, Mashayekhi M, Rommereim LM, Fox BA, Bzik DJ, Taylor GA, Turk BE, Lichti CF, et al. Phosphorylation of immunity-related GTPases by a Toxoplasma gondii-secreted kinase promotes macrophage survival and virulence. Cell Host Microbe. 2010;8(6):484-95.

39. Heitlinger E, Spork S, Lucius R, Dieterich C. The genome of Eimeria falciformis-reduction and specialization in a single host apicomplexan parasite. BMC Genomics. 2014;15:696.

40. Talevich E, Kannan N. Structural and evolutionary adaptation of rhoptry kinases and pseudokinases, a family of coccidian virulence factors. BMC Evol Biol. 2013;13:117.

41. Morris MT, Carruthers VB. Identification and partial characterization of a second Kazal inhibitor in Toxoplasma gondii. Mol Biochem Parasitol. 2003;128(1):119-22.

42. Balaji S, Babu MM, lyer LM, Aravind L. Discovery of the principal specific transcription factors of Apicomplexa and their implication for the evolution of the AP2-integrase DNA binding domains. Nucleic Acids Res. 2005;33(13):3994-4006.

43. Oberstaller J, Pumpalova Y, Schieler A, Llinas M, Kissinger JC. The Cryptosporidium parvum ApiAP2 gene family: insights into the evolution of apicomplexan AP2 regulatory systems. Nucleic Acids Res. 2014;42(13):8271-84.

44. Schmatz DM, Baginsky WF, Turner MJ. Evidence for and characterization of a mannitol cycle in Eimeria tenella. Mol Biochem Parasitol. 1989;32(2-3):263-70.

45. Schmatz DM. The mannitol cycle in Eimeria. Parasitology. 1997;114(Suppl):S81-89.

46. Samuelson J, Robbins PW. Effects of N-glycan precursor length diversity on quality control of protein folding and on protein glycosylation. Semin Cell Dev Biol. 2015;41:121-8.

47. Lorenzi H, Khan A, Behnke MS, Namasivayam S, Swapna LS, Hadjithomas M, Karamycheva S, Pinney D, Brunk BP, Ajioka JW, et al. Local admixture of amplified and diversified secreted pathogenesis determinants shapes mosaic Toxoplasma gondii genomes. Nat Commun. 2016;7:10147.

48. Wasmuth JD, Pszenny V, Haile S, Jansen EM, Gast AT, Sher A, Boyle JP, Boulanger MJ, Parkinson J, Grigg ME: Integrated bioinformatic and targeted deletion analyses of the SRS gene superfamily identify SRS29C as a negative regulator of Toxoplasma virulence. MBio 2012:3(6);e00321-12.

49. Zhou Y, Lv B, Wang Q, Wang R, Jian F, Zhang L, Ning C, Fu K, Wang Y, Qi M, et al. Prevalence and molecular characterization of Cyclospora cayetanensis, Henan, China. Emerg Infect Dis. 2011;17(10):1887-90.

50. Arrowood MJ, Donaldson K. Improved purification methods for calf-derived Cryptosporidium parvum oocysts using discontinuous sucrose and cesium chloride gradients. J Eukaryot Microbiol. 1996;43(5):89S

51. Altschul SF, Gish W, Miller W, Myers EW, Lipman DJ. Basic local alignment search tool. J Mol Biol. 1990;215(3):403-10.

52. Simao FA, Waterhouse RM, loannidis P, Kriventseva EV, Zdobnov EM. BUSCO: assessing genome assembly and annotation completeness with single-copy orthologs. Bioinformatics. 2015;31(19):3210-2.

53. Ellinghaus $D$, Kurtz $S$, Willhoeft U. LTRharvest, an efficient and flexible software for de novo detection of LTR retrotransposons. BMC Bioinformatics. 2008;9:18.

54. Krzywinski M, Schein J, Birol I, Connors J, Gascoyne R, Horsman D, Jones SJ, Marra MA. Circos: an information aesthetic for comparative genomics. Genome Res. 2009;19(9):1639-45.

55. Finn RD, Bateman A, Clements J, Coggill P, Eberhardt RY, Eddy SR, Heger A, Hetherington K, Holm L, Mistry J, et al. Pfam: the protein families database. Nucleic Acids Res. 2014:42(Database issue):D222-230.

56. Larkin MA, Blackshields G, Brown NP, Chenna R, McGettigan PA, McWilliam H, Valentin F, Wallace IM, Wilm A, Lopez R, et al. Clustal W and Clustal X version 2. 0. Bioinformatics. 2007;23(21):2947-8

57. Tamura K, Stecher G, Peterson D, Filipski A, Kumar S. MEGA6: Molecular evolutionary genetics analysis version 6.0. Mol Biol Evol. 2013;30(12):2725-9.

58. Lowe TM, Eddy SR. tRNAscan-SE: a program for improved detection of transfer RNA genes in genomic sequence. Nucleic Acids Res. 1997;25(5):955-64.

59. Laslett D, Canback B. ARAGORN, a program to detect tRNA genes and tmRNA genes in nucleotide sequences. Nucleic Acids Res. 2004;32(1):11-6. 
60. Lagesen K, Hallin P, Rodland EA, Staerfeldt HH, Rognes T, Ussery DW. RNAmmer: consistent and rapid annotation of ribosomal RNA genes. Nucleic Acids Res. 2007;35(9):3100-8.

61. Stanke M, Steinkamp R, Waack S, Morgenstern B. AUGUSTUS: a web server for gene finding in eukaryotes. Nucleic Acids Res. 2004;32(Web Server issue):W309-312.

62. Korf I. Gene finding in novel genomes. BMC Bioinformatics. 2004;5:59.

63. Lomsadze A, Ter-Hovhannisyan V, Chernoff YO, Borodovsky M. Gene identification in novel eukaryotic genomes by self-training algorithm. Nucleic Acids Res. 2005;33(20):6494-506.

64. Petersen TN, Brunak S, von Heijne G, Nielsen H. SignalP 4.0: discriminating signal peptides from transmembrane regions. Nat Methods. 2011;8(10):785-6.

65. Krogh A, Larsson B, von Heijne G, Sonnhammer EL. Predicting transmembrane protein topology with a hidden Markov model: application to complete genomes. J Mol Biol. 2001;305(3):567-80.

66. Cilingir G, Broschat SL, Lau AO. ApicoAP: the first computational model for identifying apicoplast-targeted proteins in multiple species of Apicomplexa. PLoS One. 2012;7(5):e36598.

67. Fankhauser N, Maser P. Identification of GPI anchor attachment signals by a Kohonen self-organizing map. Bioinformatics. 2005;21(9):1846-52.

68. Moriya Y, Itoh M, Okuda S, Yoshizawa AC, Kanehisa M. KAAS: an automatic genome annotation and pathway reconstruction server. Nucleic Acids Res. 2007;35(Web Server issue):W182-185.

69. Li L, Stoeckert Jr CJ, Roos DS. OrthoMCL: identification of ortholog groups for eukaryotic genomes. Genome Res. 2003;13(9):2178-89.

70. Woo YH, Ansari H, Otto TD, Klinger CM, Kolisko M, Michalek J, Saxena A, Shanmugam D, Tayyrov A, Veluchamy A, et al. Chromerid genomes reveal the evolutionary path from photosynthetic algae to obligate intracellular parasites. Elife. 2015;4:e06974

71. Castresana J. Selection of conserved blocks from multiple alignments for their use in phylogenetic analysis. Mol Biol Evol. 2000;17(4):540-52.

72. Seeber F, Feagin JE, Parsons M. Chapter 9 - The apicoplast and mitochondrion of Toxoplasma gondii. In: Weiss LM, Kim K, editors. Toxoplasma gondii (Second Edition). Boston: Academic Press; 2014. p. 297-350.

\section{Submit your next manuscript to BioMed Central and we will help you at every step:}

- We accept pre-submission inquiries

- Our selector tool helps you to find the most relevant journal

- We provide round the clock customer support

- Convenient online submission

- Thorough peer review

- Inclusion in PubMed and all major indexing services

- Maximum visibility for your research

Submit your manuscript at www.biomedcentral.com/submit

) Biomed Central 